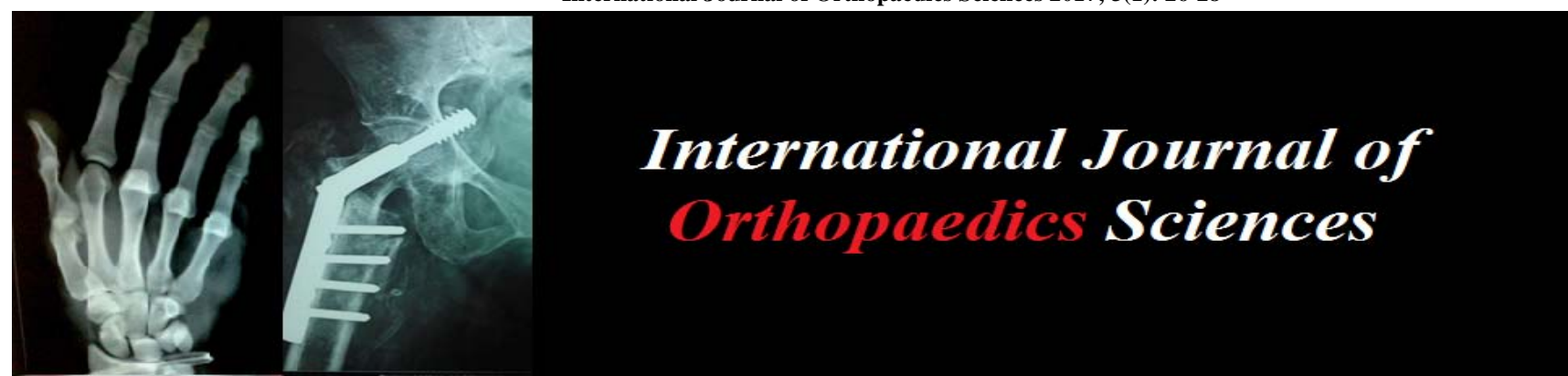

ISSN: 2395-1958

IJOS 2017; 3(1): 26-28

(C) 2017 IJOS

www.orthopaper.com

Received: 06-11-2016

Accepted: 07-12-2016

Dr. Nishantkumar Chaudhari MS (Orthopaedies),

Resident Doctor,

Department of Orthopaedic,

Surat Municipal Institute of

Medical Education and Research

(SMIMER) Surat, Gujarat, India

Dr. Rahul Parmar

MS (Orthopaedics),

Assistant Professor, Department

of Orthopaedic, Surat Municipal

Institute of Medical Education

and Research (SMIMER) Surat,

Gujarat, India

Dr. Nikhil Vachharajani

MS (Orthopaedics),

Professor and Head of Unit,

Department of Orthopaedic,

Surat Municipal Institute of

Medical Education and Research

(SMIMER) Surat, Gujarat, India

Correspondence

Dr. Nishantkumar Chaudhari

Resident Doctor, MS

ORTHOPAEDICS, Department

of Orthopaedic, Surat Municipal

Institute of Medical Education

and Research (SMIMER) Surat,

Gujarat, India

\section{Presentation of cauda equina syndrome due to pyogenic epidural abscess rare etiology of cauda equina syndrome}

\author{
Dr. Nishantkumar Chaudhari, Dr. Rahul Parmar, Dr. Nikhil Vachharajani
}

DOI: $\underline{\text { http://dx.doi.org/10.22271/ortho.2017.v3.i1a.06 }}$

\section{Abstract}

Introduction: Cauda equina syndrome is caused by compression or injury to the nerve roots distal to the level of the spinal cord. This syndrome presents as low back pain, motor and sensory deficits in the lower extremities, and bladder as well as bowel dysfunction. Acute cauda equina syndrome presenting in an immunocompetent patient is usually due to a prolapse of the intervertebral disc. Although various etiologies of cauda equina syndrome have been reported, a less common cause is infection.

Case presentation: A 35-year-old male patient presented with weakness of both lower limbs with loss of bladder and bowel control for three day. He had a history of pain in the lower back since one month. The extensors and flexors of the toes on both sides had Medical Research Council (MRC) grade of 3/5 power. Sensations were decreased below the S1 dermatome on both sides with perianal anesthesia and the ankle jerk was absent on both sides. A clinical diagnosis of cauda equina syndrome was made.

Conclusion: Cauda equina syndrome, presenting as a result of spinal infection, such as the case reported here, is extremely rare but clinically important. Surgical intervention is generally the recommended therapeutic modality with good functional outcome.

Keywords: Cauda equine syndrome, epidural abscess

\section{Introduction}

Cauda equina syndrome is caused by compression or injury to the nerve roots distal to the level of the spinal cord. This syndrome presents as low back pain, motor and sensory deficits in the lower extremities, and bladder as well as bowel dysfunction. Acute cauda equina syndrome presenting in an immunocompetent patient is usually due to a prolapse of the intervertebral disc. Although various etiologies of cauda equina syndrome have been reported, a less common cause is infection.

Vertebral osteomyelitis and epidural abscesses are most commonly caused by pyogenic organisms, particularly Staphylococcus aureus ${ }^{[1]}$. Non-pyogenic origins of such cases may be due to Mycobacterium tuberculosis or to a fungus, especially Candida or Aspergillus [2, 3]. Epidural abscess presenting as acute cauda equina syndrome in a patient has been reported not very commonly. Such cases should be treated with urgent surgical decompression and antibiotics according to sensitivity.

2. Aim: To study clinical significance and pathogenesis of unusual presentation of cauda equina syndrome.

\section{Case presentation}

A 35-year-old male patient presented with weakness of both lower limbs with loss of bladder and bowel control for three day. He had a history of pain in the lower back since one month. There was no history of cough, fever, weight loss or close contact with a patient of tuberculosis. There was no history of repeated infections or receiving corticosteroids for a prolonged duration in the recent past. The general physical examination did not reveal any abnormality. There was no localized spinal tenderness, swelling, or discharging sinus in the back. Straight leg raising tests on both sides were positive at 30 degrees. The extensors and flexors of the toes on both sides had Medical Research Council (MRC) grade of 3/5 power (i.e., the patient was able to perform the action against gravity but not against resistance). 
Sensations were decreased below the S1 dermatome on both sides with perianal anesthesia and the ankle jerk was absent on both sides. A clinical diagnosis of cauda equina syndrome was made. The routine laboratory investigations were within normal limits except $\mathrm{C}$-reactive protein (32.95, normal range 0.00 to $0.49 \mathrm{mg} / \mathrm{dL})$ and erythrocyte sedimentation rate (63, normal range 0 to $20 \mathrm{~mm} / \mathrm{hr}$ ) were elevated. Radiographs of the lumbo-sacral spine were within normal limit. An MRI of the lumbo-sacral spine showed abnormal enhancing anterior epidural collection measuring $3.5 \times 1 \mathrm{cms}$ is seen at L5/S1 level with thickening and abnormal enhancement of the adjacent meninges. (Figures 1 to 4). An ELISA test for HIV I and II antibodies was negative.

Urgent surgical decompression was planned and a laminectomy was performed at the L5-S1 levels. The epidural space contained inflammatory tissue. On pressing around disc space after retracting cauda gush of pus came out of dura from lateral aspect suggestive of intadural connection of the

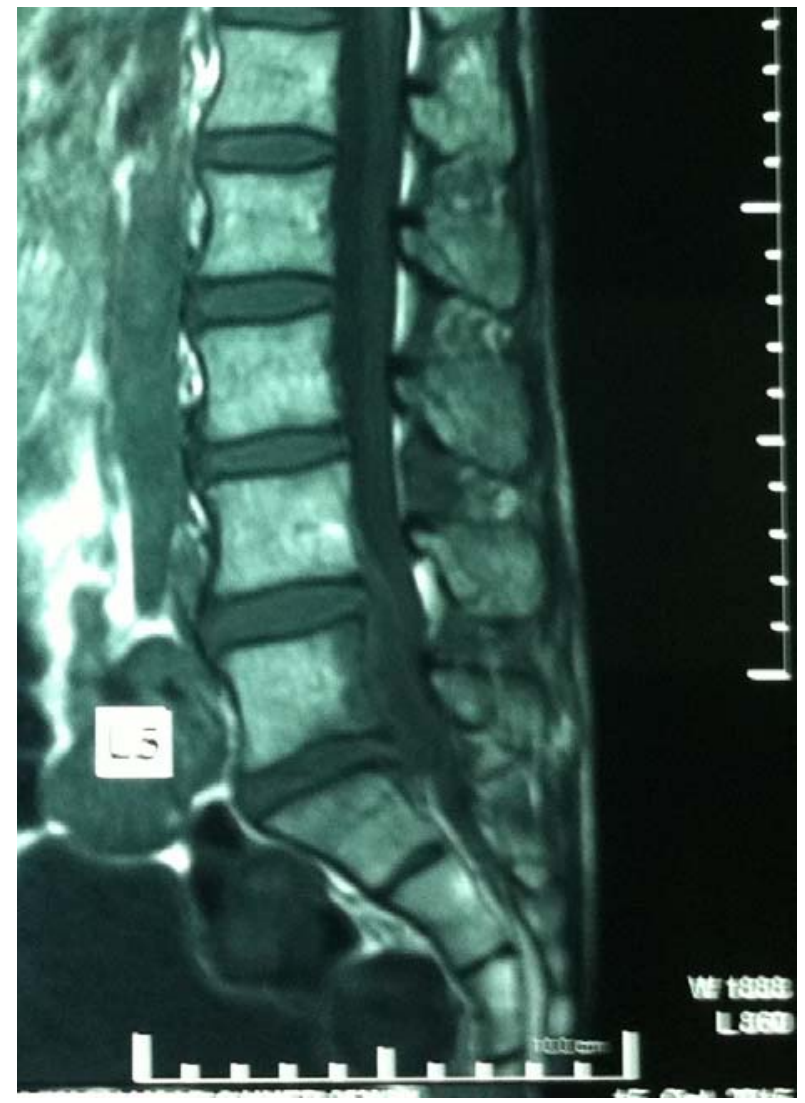

Fig 1 epidural abscess which was sent for microscopic and culture sensitivity examination. Microscopic examination showed total cell count of 150 cells per cubicmm. The culture showed growth of methicillin resistant staph aureus (MRSA). Which showed sensitivity to amikacin, gentamycin, vancomycin, teicoplanin and linezolid.

The patient was started on injectable vancomycin twice a day. Complete motor and sensory recovery was seen within a week of surgical decompression. The patient regained bladder sensations and voluntary control after 3 week of surgical decompression; however, a detailed urodynamic study was not performed. The drug was continued for a period of three weeks.

The patient was informed that the data concerning the case would be submitted for publication, and written, informed consent authorizing radiologic examination and photographic documentation was taken.

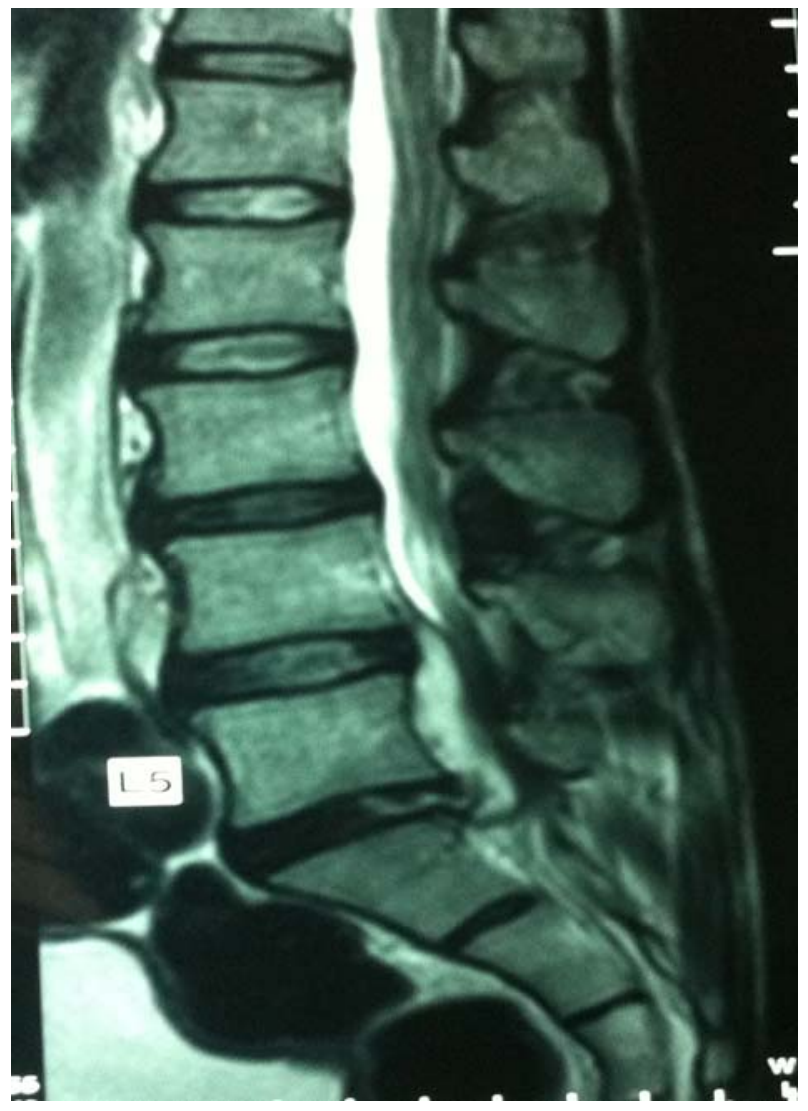

Fig 2

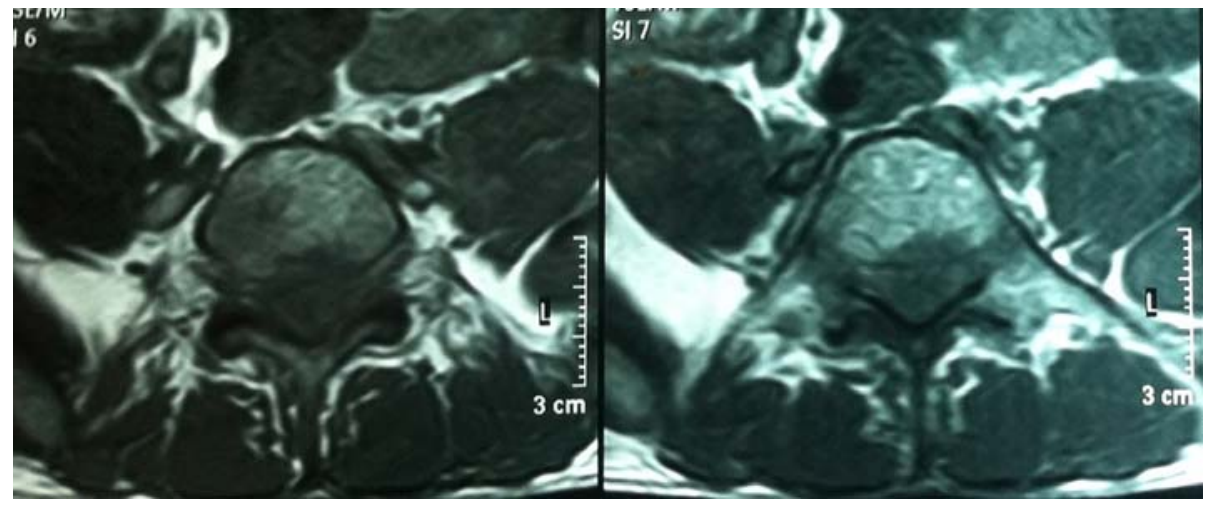

Fig 3 


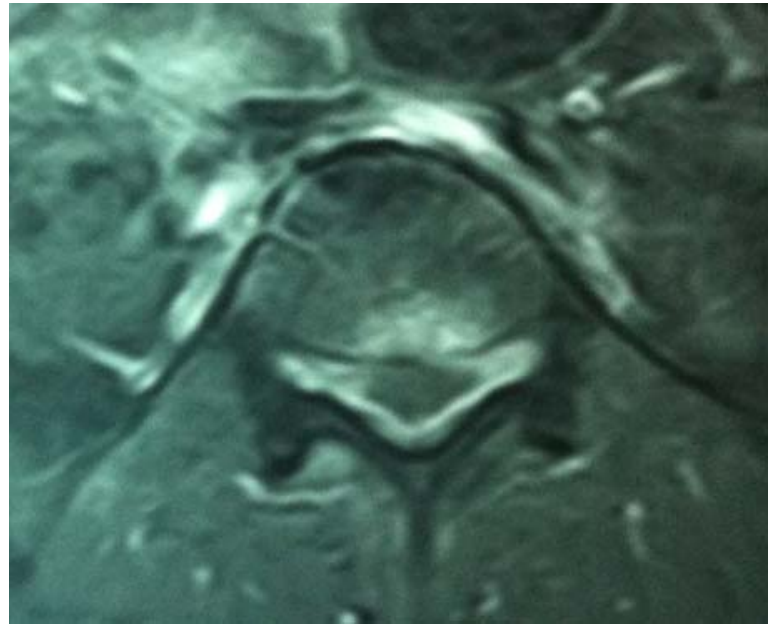

Fig 1 to 4: T1 and T2 weighted sagittal and axial MRI scans, respectively, showing abnormal enhancing anterior epidural at L5/S1 level with thickening and abnormal enhancement of the adjacent meninges.

\section{Discussion}

Cauda equina syndrome is a serious neurological condition in which there is an acute loss of function of the neurological elements of the spinal canal below the termination of the spinal cord. Cauda equina syndrome of sudden onset is regarded as a medical emergency. Surgical decompression by means of laminectomy or other approaches may be undertaken on an urgent basis if a compressive lesion, e.g. prolapsed disc, epidural abscess, tumor or hematoma is demonstrated ${ }^{[4,5]}$.

A prolapsed intervertebral disc is a common cause of cauda equina syndrome wherein the patient presents with history of backache and sudden onset of weakness with bladder and bowel involvement. ${ }^{4}$ Infective pathology with epidural collection as a cause of sudden onset cauda equina syndrome is uncommon, especially when the patient does not have any history of fever, weight loss or features of an immunocompromised status ${ }^{[5]}$. Spinal tuberculosis is a common cause of epidural abscess in a country such as India where tuberculosis is highly prevalent ${ }^{[6]}$. Fungal infection presenting with epidural collection can also be considered as a rare possibility. It is difficult to distinguish between fungal infection and tuberculosis both clinically and radiologically ${ }^{[6,}$ 7] hence a laboratory diagnosis is important to start appropriate therapy ${ }^{[8]}$.

Pyogenic spinal epidural abscesses are a rare but clinically important entity with an overall incidence of between 0.2 and 1.2 cases $/ 10,000$ hospital admissions. The mean age at presentation ranges from 50 to 60 years, and men are twice as likely to harbour the disease than women $(\sim 2: 1$ male/female ratio). Most patients who develop a spinal epidural abscess are immunocompromised due to chronic disease $(50-60 \%$ of patients), have a history of intravenous drug abuse ( $\sim 35 \%$ of patients), or have undergone spinal surgery (10-20\% of patients) ${ }^{[9,10]}$.

Staphylococcus aureus remains the most common pathogen in pyogenic spinal epidural abscesses, occurring in between 25 and $60 \%$ of patients. During the past decade, however, antibiotic-resistant organisms as well as an increase in intravenous drug abuse have revealed many new pathogens. Methicillin-resistant $S$. aureus now accounts for between 15 and $20 \%$ of pathogens in spinal epidural abscess, with gramnegative pathogens such as Pseudomonas and Escherichia coli becoming more common as well ${ }^{[11,12]}$.
The mainstay of treatment for pyogenic spinal epidural abscess is surgical decompression of the thecal sac with drainage of the abscess and long-term antibiotic therapy. The surgical approach for decompression should be modified based on particular characteristics of the case including the location of the infection (anterior compared with posterior), the region of the spine involved (cervical, thoracic, or lumbar), the presence of a significant bone or paraspinal abscess, and the degree of bone destruction revealed on CT scanning ${ }^{[11,12]}$.

Different therapeutic modalities have been proposed for the treatment of spinal osteomyelitis and epidural abscess. Although it has been primarily treated medically, certain cases may require surgical intervention. It has been suggested that, when the only symptom is back pain without significant instability or neural compression, medical treatment alone is sufficient. When spinal instability or symptoms of spinal cord or radicular compression are present, surgical decompression is indicated ${ }^{[13]}$. Our case presented with acute cauda equina syndrome and urgent decompression was required to prevent irreversible damage to the neural elements.

\section{Conclusion}

Cauda equina syndrome, presenting as a result of spinal infection, such as the case reported here, is extremely rare but clinically important. Surgical intervention is generally the recommended therapeutic modality with good functional outcome.

\section{References}

1. Sklar EML, Post MJD, Lebwohl NH. Imaging of infection of the lumbosacral spine. Neuroimaging Clin North Am. 1993; 3:577-590.

2. Ferra C, Doebbeling BN, Hollis RJ, Pfaller MA, Lee CK, Gingrich RD. Candida tropicalis vertebral osteomyelitis: a late sequela of fungemia. Clin Infect Dis. 1994; 19:697703.

3. Seligsohn R, Rippon JW, Lerner SA. Aspergillus terreus osteomyelitis. Arch Intern Med. 1977; 137:918-920.

4. Small SA, Perron AD, Brady WJ. Orthopedic pitfalls: cauda equina syndrome. Am J Emerg Med. 2005; 23:159163.

5. Cohen DB. Infectious origins of cauda equina syndrome. Neurosurg Focus. 2004; 16:e2.

6. Tuli SM. Tuberculosis of the spine. In: Tuli SM, editors. Tuberculosis of the skeletal system. 3rdedition. New Delhi: Jay pee Brothers Medical. 2004, 191-344.

7. Williams RL, Fukui MB, Meltzer CC, Swarnkar A, Johnson DW, Welch W. Fungal spinal osteomyelitis in the immunocompromised patient: MR findings in three cases. Am J Neuroradiol. 1999; 20:381-385.

8. Govender S, Rajoo R, Goga IE, Charles RW. Aspergillus osteomyelitis of the spine. Spine. 1991; 16:746-749.

9. Hakin RN, Burt AA, Cook JB: Acute spinal epidural abscess. Paraplegia, 1979; 17:330-336.

10. Rigamonti D, Liem L, Sampath $\mathrm{P}$ et al. Spinal epidural abscess: contemporary trends in etiology, evaluation, and management. Surg Neurol. 1999: 52:189-197,

11. Nussbaum ES, Rigamonti D, Standiford H et al. Spinal epidural abscess: a report of 40 cases and review. Surg Neurol. 1992; 38:225-231

12. Reihsaus E, Waldbaur H, Seeling W: Spinal epidural abscess: a meta-analysis of 915 patients. Neurosurg Rev. 2000; 23:175-205.

13. Vinas FC, King PK, Diaz FG. Spinal Aspergillus osteomyelitis. Clin Infect Dis. 1999; 28:1223-1229. 